\title{
Estudos qualitativos sobre caminhoneiros e HIV/aids: contribuições para análise de vulnerabilidade
}

\author{
Qualitative studies on truck drivers and HIV/AIDS: \\ contributions for the analysis of vulnerability
}

${ }^{1}$ Departamento de Ciências da Vida, Universidade do Estado da Bahia. R. Silveira Martins 2555, Cabula. 41150-000 Salvador BA Brasil. laiomagnoss@gmail.com

\begin{abstract}
The scope of this article is to conduct a critical identification and analysis, based on the analytical framework of the concept of vulnerability of truck drivers to HIV. The criteria for inclusion were to address the issue of truck drivers' susceptibility to HIV/AIDS and to adopt the qualitative approach. A total of 445 abstracts were located, of which 17 articles were included in the analysis and categorized as "sociocultural studies", "evaluative studies" and "risk behavior studies." The analysis was based on reflections surrounding the concept of vulnerability in health. The study criticizes the predominance of qualitative studies of a behaviorist nature, with an emphasis on the identification of risk behaviors, concepts and representations about HIV/AIDS. Furthermore, it points to studies of a sociocultural and evaluative nature that transcend the barrier of individual behaviors, expanding the scope of analysis, anal$y$ sis of structural phenomena and interactions of subjects faced with the epidemic, duly approaching the concept of vulnerability. The review reveals the need for studies that take into account the concept of vulnerability, contextualizing the behaviors to the socio-structural dimensions involved in the AIDS epidemic.
\end{abstract}

Key words Truck drivers, Health vulnerability, Acquired Immunodeficiency Syndrome, HIV
Resumo $O$ artigo pretende identificar e analisar criticamente, com base no quadro analítico do conceito de vulnerabilidade, estudos qualitativos sobre a vulnerabilidade de caminhoneiros ao HIV. Os critérios de inclusão foram: abordar a temática da susceptibilidade dos caminhoneiros ao HIV/ AIDS e empregar a abordagem qualitativa. Foram encontrados 445 resumos, dentre os quais 17 artigos foram incluídos na análise e categorizados em "estudos socioculturais", "estudos avaliativos" e "estudos de comportamentos de risco". A análise foi balizada por reflexões realizadas a partir do conceito de vulnerabilidade em saúde. O estudo critica a predominância de estudos qualitativos de cunho comportamentalista, com ênfase na identificação de comportamentos de risco, e de concepções e representações sobre HIVIAIDS. Além disso, aponta para estudos de matriz sociocultural e avaliativos que transpassam a barreira dos comportamentos individuais, ampliando o escopo de análise, ao compreender os fenômenos estruturais e interações dos sujeitos frente à epidemia, aproximando-se do conceito de vulnerabilidade. A revisão aponta para a necessidade de estudos que levem em consideração o conceito de vulnerabilidade, contextualizando os comportamentos às dimensões socioestruturais envolvidas na epidemia de aids.

Palavras-chave Caminhoneiros, Vulnerabilidade em saúde, Sindrome da Imunodeficiência Adquirida, HIV 


\section{Introdução}

Em todo o planeta, estima-se cerca 36,7 milhões de pessoas vivendo com HIV/aids (PVHA) ${ }^{1}$, sendo que no Brasil, em 2014, a estimativa foi de 781 mil PVHA, o que corresponde a uma prevalência de $0,39 \%$ na população em geral. Entretanto, estima-se que ainda $17 \%$ deste total de PVHA ainda não sabem do seu status sorológico ${ }^{2}$. Para avançar na melhoria deste quadro epidemiológico, o Programa Conjunto das Nações Unidas sobre HIV/ AIDS (UNAIDS) articulou três metas para 2020: 90\% de todas as PVHA tendo conhecimento de seu estado sorológico; $90 \%$ de todas as PVHA recebendo terapia antirretroviral (TARV); e, por fim, $90 \%$ de todas as PVHA e recebendo a TARV, tendo supressão viral ${ }^{3}$.

Estas metas são ambiciosas e terão pela frente desafios complexos, como o acesso à prevenção e ao tratamento do HIV, o enfrentamento do estigma e da criminalização de pessoas LGBT e a maior atenção aos grupos sociais em contextos de maior vulnerabilidade ${ }^{4}$ (denominados, atualmente, de populações-chave para epidemia). Além disso, um desafio teórico se redesenha através da atual e necessária reflexão sobre os contextos de vulnerabilidade em que as pessoas estão inseridas e a ideia de comunidade (noções que foram muito importantes para as respostas iniciais bem sucedidas à epidemia $)^{5-7}$.

Apesar de todos serem considerados vulneráveis ao HIV/aids, alguns grupos sociais merecem uma atenção específica das ações de saúde, pois vários estudos têm mostrado que o processo de disseminação da epidemia, seu impacto e seus determinantes são desiguais entre as populações. O reconhecimento destas especificidades, a partir de uma perspectiva do conceito de vulnerabilidade, não objetiva conformação de "grupos de risco", mas sim a compreensão de fenômenos sociais para o planejamento e a implementação de políticas de saúde voltadas para o fortalecimento dos direitos ${ }^{8}$.

Nesse sentido, a população de caminhoneiros tem sido apontada em diferentes regiões do mundo como uma população com maior risco de adquirir o HIV, devido a algumas características laborais peculiares, como a mobilidade por rotas de longa distância e não permanência em suas cidades de origem ${ }^{3}$, principalmente em regiões do continente africano ${ }^{9-12}$ e asiático $^{13-16}$.

Estudos brasileiros também têm se atentado para esta dinâmica peculiar, e apontam para fatores e questões relacionadas à vulnerabilidade de caminhoneiros ao HIV/aids ${ }^{17-22}$. Comumen- te, os caminhoneiros são descritos na literatura como indivíduos que se relacionam com parceiras eventuais durante as viagens de longa distância $^{9,15,17,18,22}$. Estudos também têm apontado para existência de fatores, significados e concepções que apoiam a não utilização do preservativo nas relações sexuais desses sujeitos com suas parceiras $^{12,15,16,22}$. De acordo com Leal ${ }^{18}$, muitos dos estudos com esta população foram orientados pela metodologia de aferição de Conhecimentos, Atitudes e Práticas (chamados de CAP, ou KAP em Inglês), que possuem um enquadramento teórico-analítico orientado para o risco.

Numa outra perspectiva, mais estrutural, estudos realizados no Brasil têm relatado que os caminhoneiros vivenciam uma dinâmica de trabalho intenso nas rotas de longa distância ${ }^{23-25}$. Estas rotas podem ser espaços que submetem esses sujeitos a uma diversidade de situações e relações que podem incidir no processo de vulnerabilidade ao HIV, pois os fazem vivenciar condições precárias relacionadas ao trabalho desenvolvido (ambiente precário, pressões de transportadoras etc.), sem o suporte social próximo (família, amigos etc.) e sob a pena de estarem muitos dias de suas vidas como migrantes entre várias cidades $\mathrm{e}$ visitantes no espaço familiar.

Nesse sentido, o presente artigo pretende identificar e analisar criticamente os estudos qualitativos publicados sobre a vulnerabilidade de caminhoneiros ao HIV, com base no quadro analítico do conceito de vulnerabilidade.

\section{Metodologia}

Trata-se de um estudo de revisão integrativa da literatura ${ }^{26}$. Seis etapas ${ }^{27}$ foram utilizadas para a construção desta revisão, a saber: identificação da questão norteadora da pesquisa, estabelecimento de critérios de inclusão/exclusão de artigos, definição de informações a serem extraídas dos artigos selecionados, análise dos dados, interpretação dos resultados e apresentação da revisão. Também foi utilizado o guideline PRISMA para sistematizar o processo de pesquisa da presente revisão $0^{28}$.

$O$ estudo apresentou a seguinte questão norteadora: quais as evidências disponíveis nos estudos qualitativos sobre a vulnerabilidade de caminhoneiros ao HIV/aids para o quadro analítico do conceito de vulnerabilidade? A coleta dos dados foi realizada no mês de setembro de 2016 na base de dados do PubMed, Biblioteca Virtual em Saúde (BVS - composta por diversas bases de dados, como por exemplo, LILACS e MEDLINE, 
para mais informações: http://bvsalud.org/sobre -o-portal/), Web of Science e Scopus. A busca foi feita através da utilização das seguintes palavraschave: truck, drivers, hiv, aids.

Os critérios de inclusão no estudo foram os seguintes: abordar a temática da vulnerabilidade dos caminhoneiros ao HIV/aids e empregar na seção de metodologia a abordagem qualitativa para produção de dados (entrevista em profundidade, entrevista semi-estruturada, grupo focal, entrevista narrativa, observação participante). Já os critérios de exclusão foram: não ter tido acesso ao artigo completo e estar escrito em outra língua diferente do inglês, português e espanhol.

Para coleta de dados foi utilizada uma planilha do Microsoft Excel ${ }^{\circledR}$ contendo os seguintes termos: palavra-chave utilizada na busca, base de dados, número total de artigos encontrados, nome do periódico, título do artigo, autores, ano de publicação, país de publicação e resumo. Inicialmente, realizou-se a leitura de todos os resumos que foram selecionados na busca através das palavras-chave. A exclusão dos artigos da análise foi realizada durante a leitura dos resumos de acordo com a aplicação dos critérios supracitados. Os estudos com métodos mistos (quantitativo e qualitativo) também foram incluídos.

Na base de dados PubMed foi identificado um total de 145 resumos e, a partir da leitura destes e da aplicação dos critérios de inclusão, foram excluídos 131; após a leitura dos 14 artigos que foram selecionados, um deles foi excluído da análise porque não tinha os caminhoneiros como sujeitos do estudo; no final, incluiu-se 13 artigos elegíveis desta base de dados para análise. Na BVS foram identificados 56 resumos, 50 foram excluídos depois da leitura dos resumos, 5 foram excluídos por estarem repetidos na base PubMed e apenas um foi selecionado.

Na base de dados Web of Science foram identificados 134 resumos, sendo que 120 foram excluídos após leitura dos resumos, enquanto 10 foram excluídos por estarem repetidos na $P u b$ Med. Durante a leitura dos artigos, um deles foi excluído porque, apesar de ter sido identificado no resumo como uma etnografia, o estudo apresentou somente dados de um survey; no final, foram elegíveis três artigos nesta base. Na base Scopus não foram encontrados artigos elegíveis, pois todos os resumos que poderiam ser incluídos estavam repetidos em outras bases de dados. Finalmente, foram incluídos 17 artigos na análise (Figura 1).
Realizou-se a leitura dos artigos na íntegra e a análise de conteúdo foi utilizada para a categorização. Posteriormente, procedeu-se a categorização em três grupos temáticos: "estudos socioculturais"29-31, "estudos avaliativos"32-36 e "estudos de comportamentos de risco"19,37-44. Os artigos que foram classificados como "estudos de comportamento de risco" foram marcados pela ênfase nos seguintes temas: quantidade de parceiras sexuais, utilização dos serviços de profissionais do sexo, consumo de álcool e outras drogas, utilização e percepções acerca do preservativo, percepções de risco, práticas sexuais de risco, conhecimentos e representações sociais sobre o HIV/aids.

Os "estudos avaliativos" foram classificados desta maneira por darem ênfase à avaliação de determinadas tecnologias preventivas, como por exemplo, a Profilaxia Pré-Exposição (PrEP), a circuncisão e materiais de campanha. Já os estudos socioculturais foram abordagens baseadas em narrativas dos sujeitos e na descrição densa de elementos culturais de contextos locais, que tinham como objetivo a compreensão de relações intersubjetivas e fenômenos estruturais do processo de vulnerabilidade, sem se limitar estritamente à noção de comportamentos de risco.

A análise crítica da produção dos dados foi realizada através do conceito de vulnerabilidade trabalhado pelos autores Mann ${ }^{45}$ e Ayres et al. ${ }^{7}$.

\section{Resultados e Discussão}

Os artigos analisados foram organizados no Quadro 1, que contempla as seguintes informações: objetivos, autores, periódico/base de dados, ano de publicação, técnicas de produção de dados qualitativos e principais resultados. Estes artigos foram publicados em periódicos diferentes, com destaque para duas publicações na Aids Care $^{19,29}$. Ao analisar a classificação dos periódicos no portal de revistas da BVS (http://portal. revistas.bvs.br/index.php?lang=pt), verificou-se que todas elas foram classificadas primeiramente na área temática das ciências da saúde, exceto a Social Science and Medicine ${ }^{37}$, que esteve alocada na área das ciências humanas.

Os países em que foram realizadas estas pesquisas são caracterizados pela intensa desigualdade social e pobreza. No presente estudo, o continente africano, mais especificamente a África subsaariana, teve o maior número de pesquisas realizadas, seguido da Ásia e América Latina (2 estudos realizados no Brasil). 


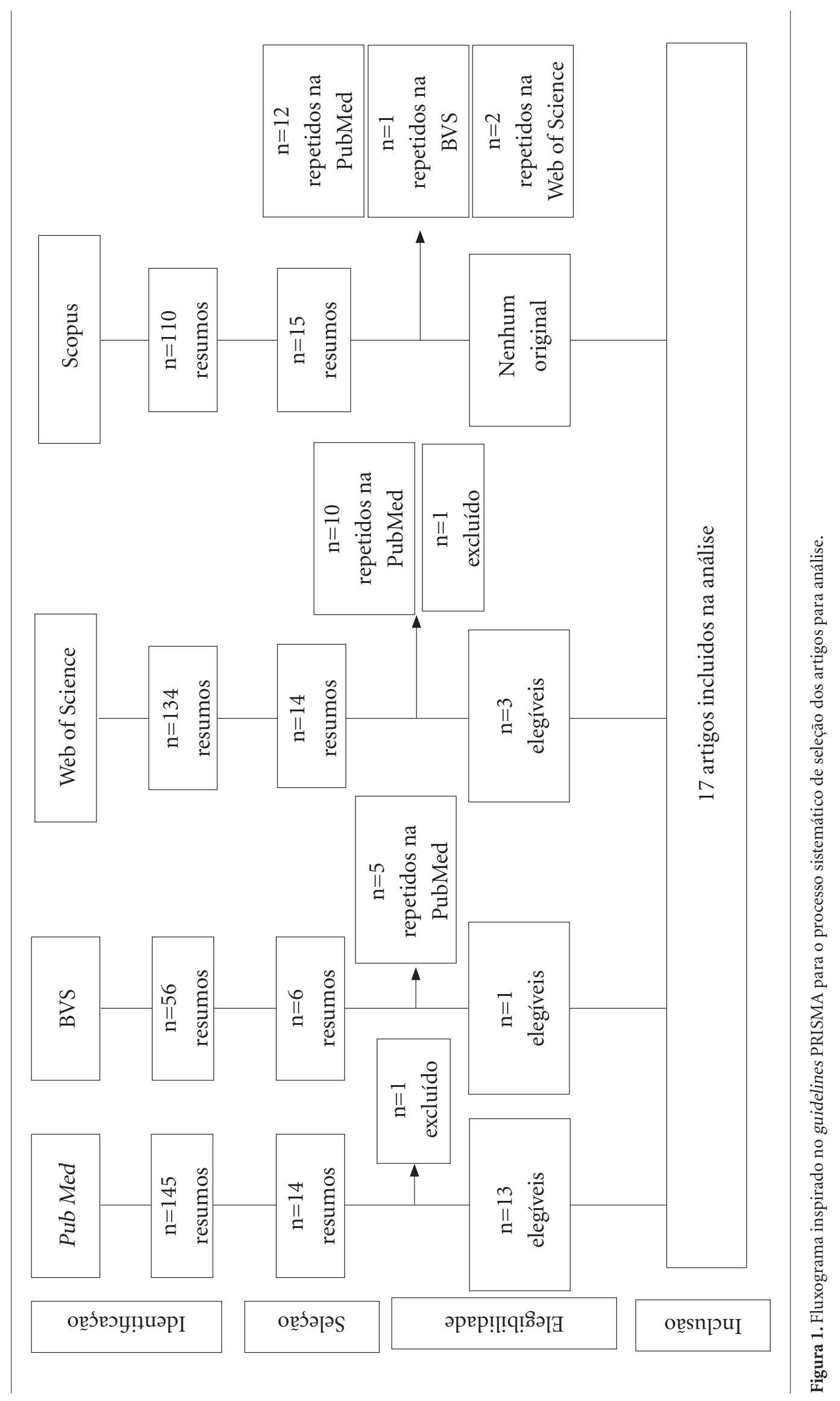




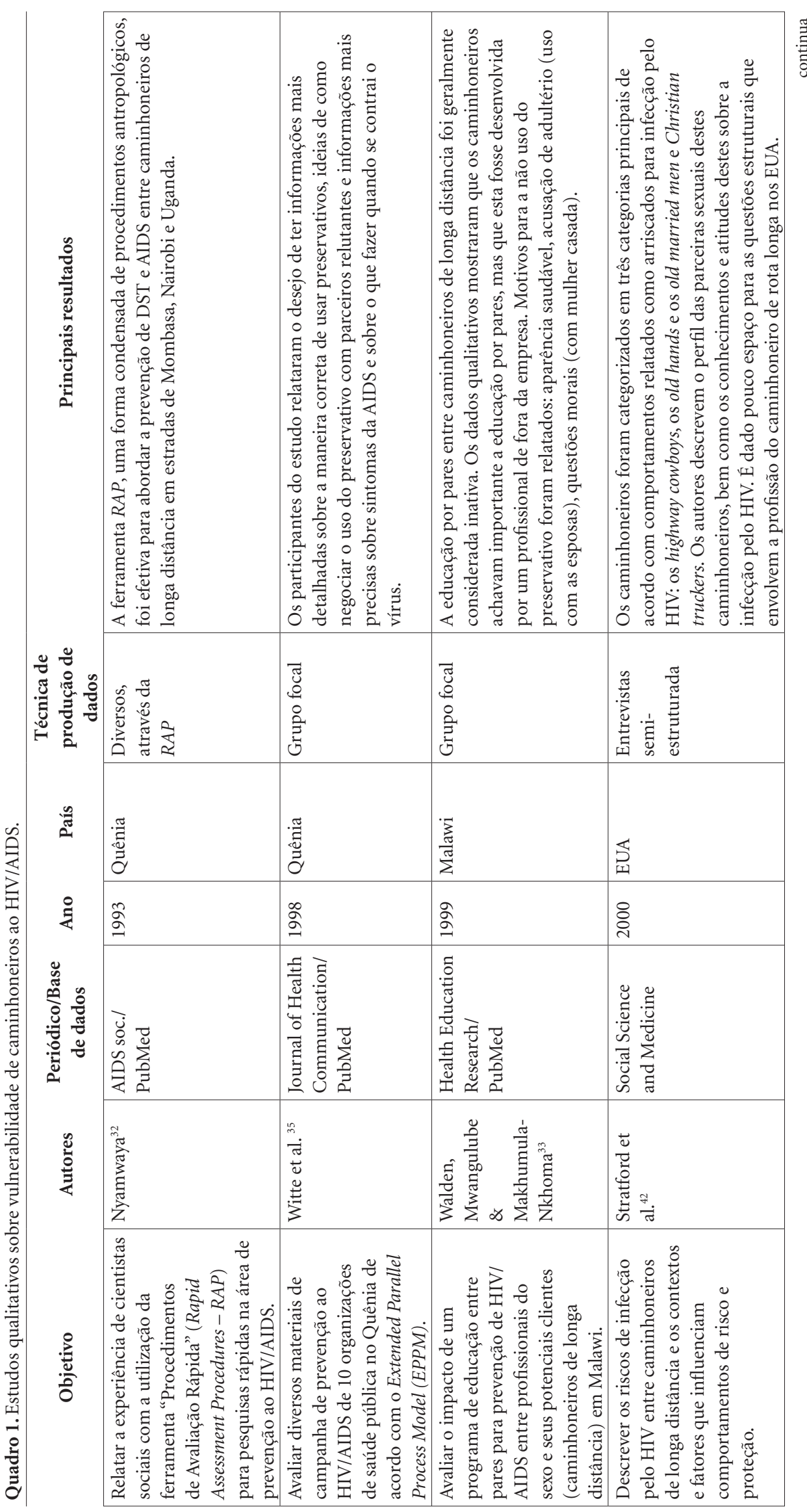




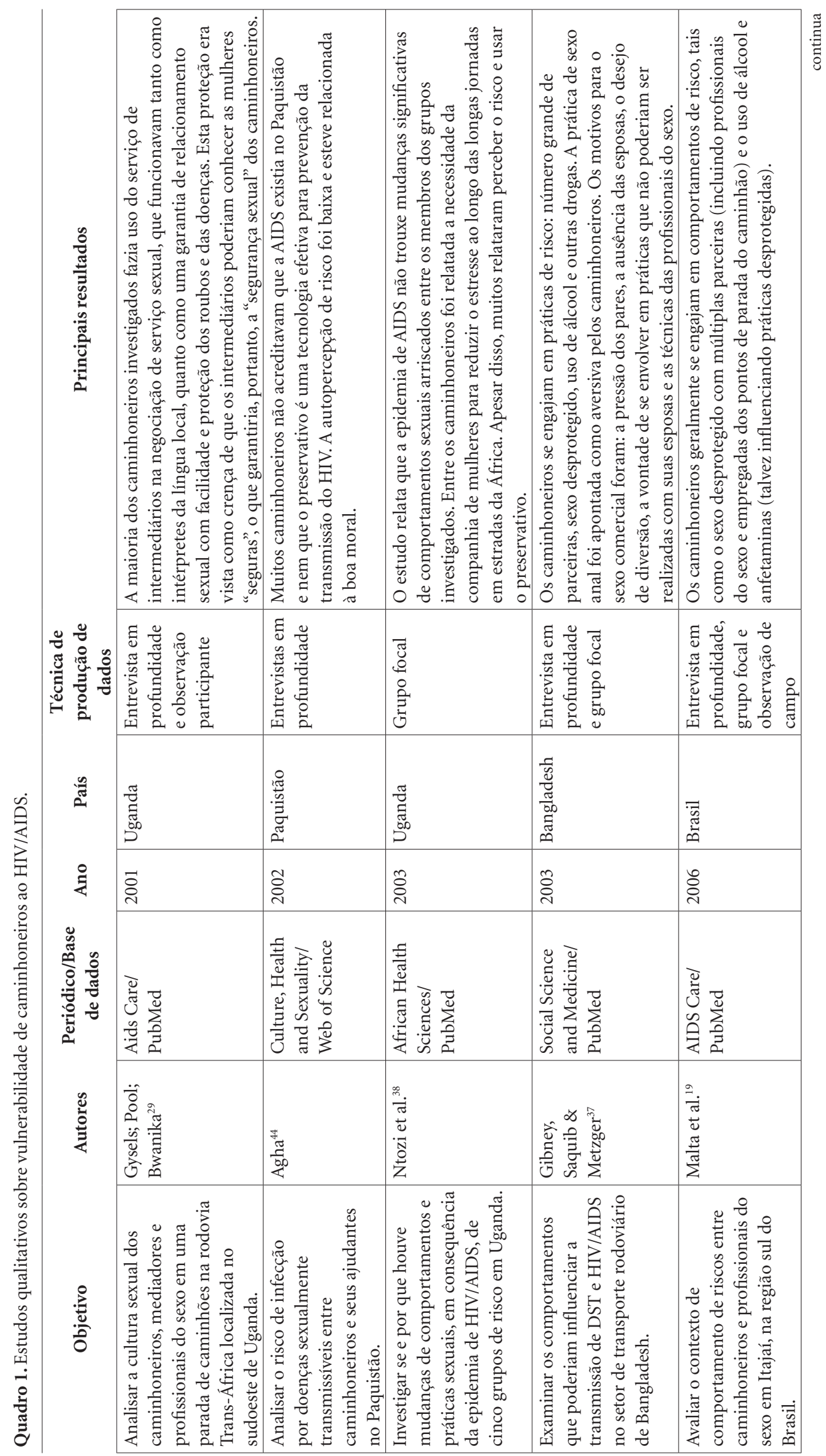




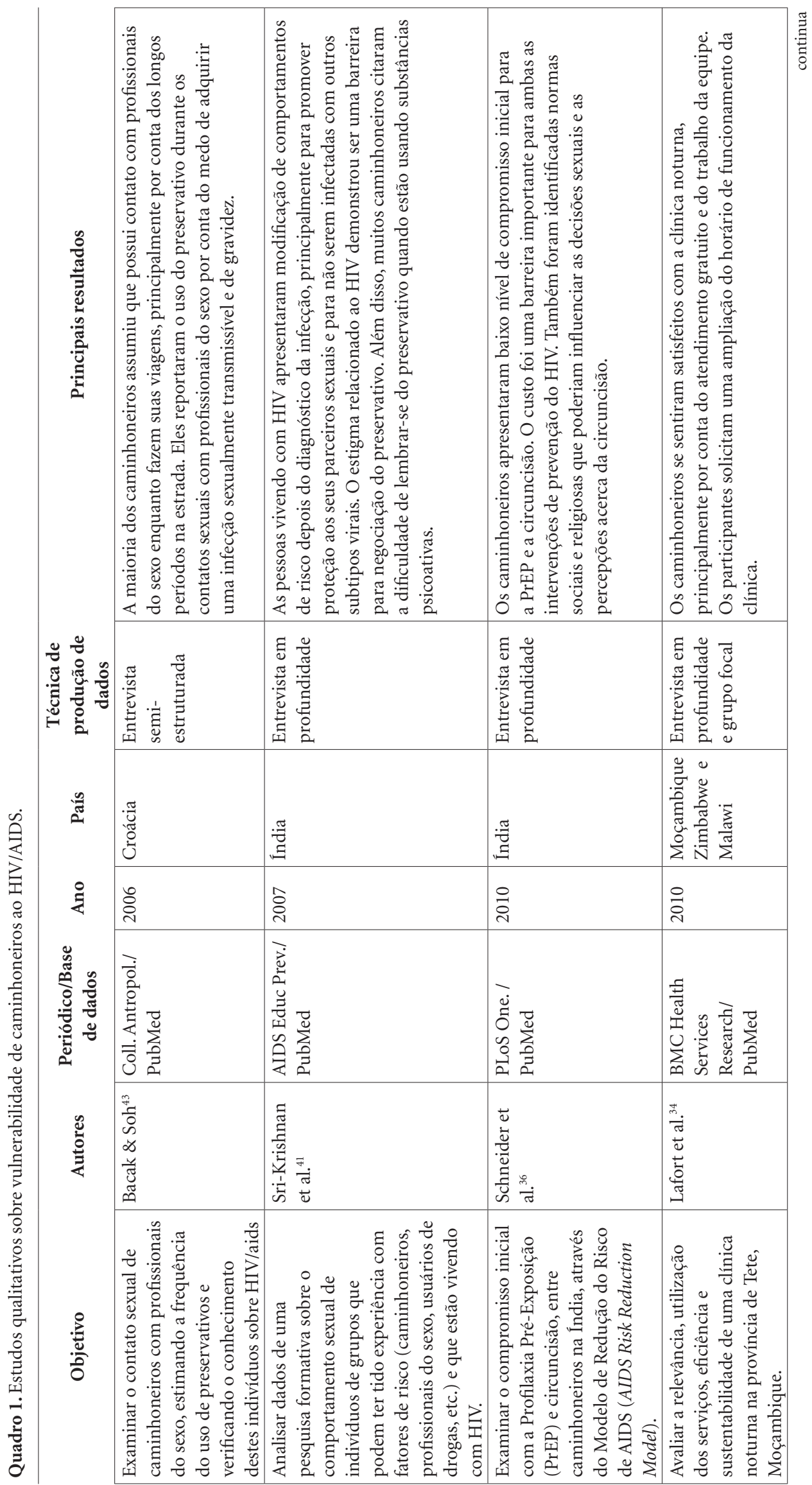


골

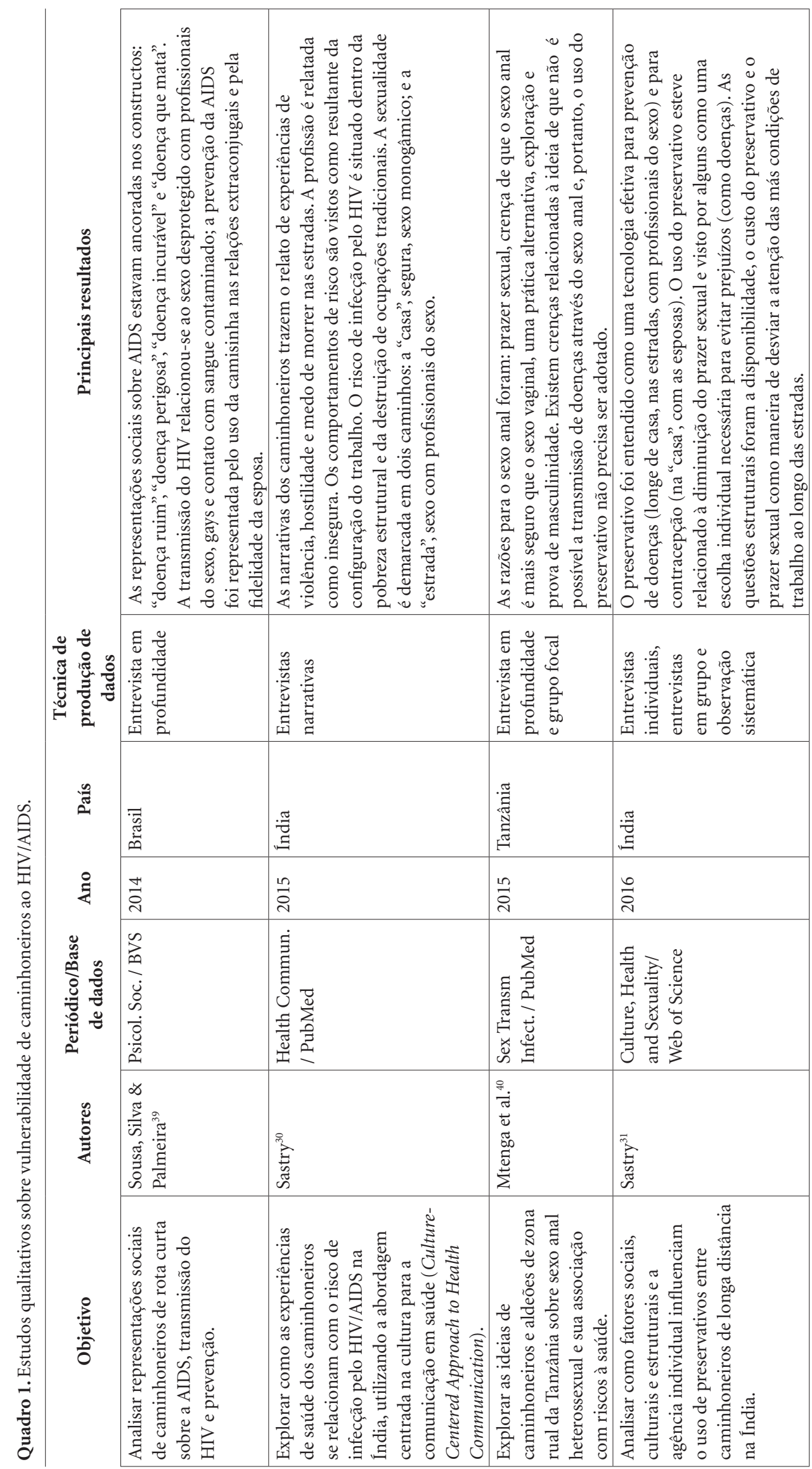


Ressalta-se que apesar destes países possuírem baixos valores do Índice de Desenvolvimento Humano (menor que 0,57, exceto o Brasil com 0,75 e a Índia com 0.60 ), o contexto da pobreza e desigualdade social não foi destacado na maior parcela dos estudos. Dados oriundos da United Nations Development Programme, 2014 (http:// hdr.undp.org/en/composite/HDI)

Embora diversos estudos internacionais indiquem que fatores estruturais podem facilitar a transmissão do HIV e sua concentração em áreas geográficas e populações específicas ${ }^{46}$, apenas alguns estudos refletiram sobre a pobreza existente nos cenários investigados. Estes artigos trouxeram para tela a discussão das condições precárias de vida de profissionais do sexo como fatores que poderiam influenciar o sexo comercial com caminhoneiros ${ }^{29,38}$.

No que tange às abordagens metodológicas, percebe-se que a utilização de dados quantitativos e qualitativos foi marcante em quatro artigos. A integração de diferentes métodos fomenta o enriquecimento dos dados dos estudos ao promover a integração interdisciplinar entre diversas abordagens metodológicas ${ }^{47}$.

Os estudos de comportamentos de risco foram marcados pela ênfase em conhecimentos e representações sobre HIV/aids e em comportamentos, atitudes e práticas arriscadas. Vale a pena relembrar que as iniciais compreensões a respeito da epidemia de aids foram marcadas principalmente pelo enfoque biomédico, epidemiológico e comportamentalista. Mann ${ }^{45}$, em seu clássico livro Aids in The World, propõem três períodos históricos ligados às respostas dadas frente ao desafio da doença. O primeiro deles foi o período da descoberta do vírus HIV (1981-1984), que foi caracterizado principalmente pelos estudos epidemiológicos sobre a doença. Estes estudos ofereceram a descrição de informações a respeito das vias de transmissão do vírus e identificaram os comportamentos de risco associados com a infecção. Neste período, as ações em saúde pública foram direcionadas para a divulgação de informações a respeito de "grupos de risco" e "comportamentos de risco" associados à doença com o objetivo de estimular mudanças de comportamentos individuais.

A produção de estudos epidemiológicos, com base no conceito de risco, no início da epidemia de aids foi intensa, o que promoveu a identificação de subgrupos populacionais que possuíam uma maior probabilidade de se encontrar pessoas com a doença, em comparação com a população geral ${ }^{7}$.
A propagação do termo "grupo de risco" foi realizada pela mídia de massa e ganhou conotações prejudiciais para a prevenção. Ao invés de promover a prevenção, houve a promoção de preconceitos e discriminação, tornando complicada a situação social e clínica das pessoas infectadas pelo vírus ${ }^{7}$ e reforçando conotações estigmatizadoras de grupos sociais marginalizados, com destaque para os homossexuais ${ }^{48}$.

O segundo período histórico (1985-1988), denominado de período das primeiras respostas ao HIV/aids, foi balizado pela consideração de que a prevenção e o cuidado eram processos distintos e programaticamente separados. Dessa maneira, o alvo principal da atenção neste período foi a diminuição dos comportamentos de risco individuais. Para alcançá-lo foram criados diversos programas nacionais e internacionais (como o World Health Organizaton's Global Program on AIDS - WHO/GPA), que tinham como meta três elementos básicos para a redução de riscos: informação e educação, serviços sociais e de saúde e não discriminação das $\mathrm{PVHA}^{45}$.

Dessa maneira, o conceito de grupos de risco foi dando lugar ao conceito de comportamentos de risco, que ampliou o leque de possibilidades de entendimento da dinâmica da epidemia, incluindo também outros grupos sociais ao focar os comportamentos individuais ${ }^{7}$. Os principais avanços deste novo conceito foi ampliar o espectro de estratégias de prevenção para todos os indivíduos e a tentativa de mitigar o estigma entre os grupos com maior incidência da doença. Por outro lado, ao responsabilizar unicamente os indivíduos pela mudança de seus comportamentos, este conceito propiciou a "culpabilização" de pessoas infectadas ${ }^{7}$.

Em nossa revisão, apesar dos estudos analisados na categoria "estudos de comportamentos de risco" terem sido publicados nos anos 2000, observa-se que o arcabouço teórico-metodológico se ancorou na matriz teórica comportamentalista, classificada por Man e Tarantola como típica dos dois primeiros períodos da epidemia (de 1981 a 1988).

Por exemplo, o estudo realizado em Bangladesh teve como objetivo examinar os comportamentos que poderiam influenciar a transmissão de DST e HIV/aids no setor de transporte rodoviário de Bangladesh ${ }^{37}$; um dos estudos brasileiros visou avaliar o contexto de comportamentos de risco entre caminhoneiros e profissionais do sexo em Itajaí ${ }^{19}$, enquanto o outro analisou as representações sociais de caminhoneiros de rota curta sobre a AIDS e sobre a prevenção e transmissão do HIV ${ }^{39}$. 
Um estudo realizado na Tanzânia buscou explorar as ideias de caminhoneiros sobre o sexo anal heterossexual e sua associação com o risco de infecção pelo HIV ${ }^{40}$; um estudo em Uganda se propôs a investigar se houve mudanças nos comportamentos e nas práticas sexuais entre os grupos de risco depois da epidemia de aids ${ }^{38}$; um estudo no Paquistão buscou analisar o risco de infecção por doenças sexualmente transmissíveis entre caminhoneiros ${ }^{44}$; um estudo na Croácia analisou o contato sexual de caminhoneiros com profissionais do sexo com foco em comportamentos de risco e conhecimento sobre HIV/aids ${ }^{43}$.

Em 2007 foi publicada uma pesquisa formativa realizada na Índia sobre os comportamentos sexuais de pessoas (provenientes de grupos com comportamentos de risco, dentre eles, caminhoneiros) vivendo com HIV. Este estudo demonstrou a mudança de comportamentos dos indivíduos após o diagnóstico de infecção pelo HIV, mostrou diferenças importantes na negociação do preservativo de acordo com o gênero e as práticas sexuais e revelou que os caminhoneiros tinham dificuldade de usar o preservativo quando estavam sob o efeito de substâncias psicoativas ${ }^{41}$.

Semelhantemente, alguns estudos classificados aqui como "avaliativos" também demonstraram matriz teórico-conceitual comportamentalista. Walden et al. ${ }^{33}$, por exemplo, investigaram o impacto de um programa educativo de pares com caminhoneiros e profissionais do sexo em Malawi, através da mensuração quantitativa da autopercepção de risco, do conhecimento da aids e dos comportamentos de risco (uso de preservativo e sexo casual nas últimas 3 semanas) entre grupos de intervenção e controle. Neste estudo, os dados qualitativos foram utilizados para aprofundamento das razões do uso inconsistente de preservativo e para avaliar a aceitação de educação por pares.

O estudo de Nyamwaya ${ }^{32}$ destacou principalmente a ferramenta intitulada "Procedimentos de Avaliação Rápida" (Rapid Assessment Procedures - RAP), que tinha o objetivo de simplificar a pesquisa antropológica através do uso de técnicas básicas desta disciplina, tais como entrevistas, observação participante e grupos focais, para pesquisas rápidas na área de prevenção da transmissão de HIV. Para descrever a efetividade desta ferramenta, o autor focou especialmente na produção de dados sobre aspectos comportamentais arriscados de caminhoneiros africanos.

A maior parcela destes estudos comportamentalistas veem os caminhoneiros como um dado "grupo de risco" ou como uma determi- nada população que possui "comportamentos sexuais arriscados", naturalizando a vulnerabilidade como uma condição intrínseca do sujeito e não como um contexto complexo.

O estudo conduzido por Stratford et al. ${ }^{42} \mathrm{faz}$ um esforço de contextualizar o contexto socioestrutural em os caminhoneiros de rota longa nos Estados Unidos estão inseridos, porém decidimos classificá-lo como um estudo de "comportamentos de risco", pois os autores dedicam grande parte da apresentação dos resultados e da discussão às questões relacionadas aos comportamentos de risco dos sujeitos da pesquisa para infecção pelo HIV. Neste estudo, os caminhoneiros são categorizados em três categorias principais: os highway cowboys, aqueles mais jovens, com relato de uso de substâncias psicoativas e com práticas sexuais arriscadas, como o não uso do preservativo; os old hands eram mais velhos que os primeiros, geralmente casados ou divorciados, e com relato comportamentos sexuais de risco "moderados" quando comparados os primeiros, motivados principalmente pela relação com a família e com o declínio físico. Por fim, os old married men e Christian truckers relatavam um relacionamento mais estável com suas mulheres e avessos aos comportamentos de risco, motivados principalmente por crenças morais e religiosas.

A fundamentação conceitual em noções de "grupo de risco" e "comportamento de risco", que promoveu estigmatização do PVHA e restringiu as ações de prevenção, parece não ter mudado muito o tom de muitas das pesquisas publicadas. Na história da aids, pode-se observar que os movimentos sociais organizados, como os movimentos pelo direito das mulheres e homossexuais, questionaram tais categorias comportamentalistas e individualistas. Esta crítica fundamentou-se na ideia de que o uso do preservativo não se resumiria a simples decisão individual, a partir de informações e da própria vontade das pessoas, mas estaria intrinsecamente relacionado a aspectos de ordem social e interpessoal ${ }^{7}$.

O terceiro período da epidemia de aids, proposto por Man ${ }^{45}$, iniciou-se em 1989 e se estenderia até a data da publicação do texto pelos autores, e é descrita pelos autores como sendo marcada pelo conceito de vulnerabilidade. A emergência do conceito de vulnerabilidade na década de 1990 foi visto como tentativa de apreensão da predisposição à infecção através de uma perspectiva completamente diferente daquelas orientadas pelo conceito de risco ${ }^{48}$.

Este conceito aplicado ao campo da saúde foi resultado de um processo de interseções 
entre o ativismo político frente à epidemia e o movimento dos Direitos Humanos ${ }^{7}$. A vulnerabilidade coloca em cena o entendimento das conexões existentes entre o suporte para as PVHA e os esforços para diminuição da propagação da epidemia, levando em consideração as questões sociais, culturais, políticas e econômicas. Assim, a partir do marco teórico-conceitual da vulnerabilidade, dever-se-ia ocorrer o deslocamento do foco das pesquisas de questões exclusivamente individuais, tais como comportamentos, atitudes e práticas, para a atenção aos aspectos sociais, tais como o estigma, o acesso aos serviços de saúde, a pobreza etc. ${ }^{45}$.

Mann ${ }^{45}$ propõem três categorias explicativas interdependentes para a vulnerabilidade: a individual, a programática e a social. Ayres et al. ${ }^{49} \mathrm{em}$ meados dos anos 2000 fazem uma síntese conceitual ao abordar o objetivo da vulnerabilidade individual como a identificacão dos fatores físicos, mentais ou comportamentais por meio de avaliações de risco e de outras abordagens; a vulnerabilidade social como a análise das dimensões da cultura, religião, moral, política, economia e fatores institucionais pelos quais podem determinar os meios de exposição ao vírus; e a vulnerabilidade programática como a análise das políticas, dos programas e dos serviços que interferem no cotidiano social e individual das pessoas.

Em revisão conceitual mais atual, Ayres et al. ${ }^{7}$ ressaltam a necessidade de levar-se em consideração a sinergia entre estas três dimensões da vulnerabilidade com o objetivo de tornar os conceitos de prevenção e promoção da saúde "mais realistas, pragmáticos e eticamente orientados". Além disso, seria imprescindível ressaltar que a dimensão individual não se limitaria a vontade cognitiva e individual de tomada de decisões, mas que esta dimensão parte do pressuposto de que o indivíduo - ou porque não o sujeito? - é um ser "em relação". Assim, "o indivíduo é compreendido como intersubjetividade e como ativo coconstrutor, e não apenas resultado (efeito) das relações sociais, que devem, então, ser remodeladas para garantir o 'comportamento saudável"'7.

A perspectiva do conceito de vulnerabilidade trabalhada pelos autores brasileiros desloca a valorização inicial da dimensão individual, proposta pelo grupo de Mann e colaboradores, para uma reconfiguração conceitual das categorias de vulnerabilidade individual e programática, que é inspirada principalmente pela matriz teórica dos Direitos Humanos e por conceitos como o de intersubjetividades. Vale a pena ressaltar que a perspectiva brasileira trouxe muitos ganhos $e$ ampliou o escopo de análise com a incorporação de novos conceitos à reflexão em torno da vulnerabilidade. Entretanto, apesar do deslocamento apresentado, temos a hipótese de que a produção de dados empíricos nesse campo ainda confunde muito o conceito de vulnerabilidade com o conceito de risco ou reduzem a intensa dinâmica social em cisões entre a vulnerabilidade individual e coletiva.

No campo da vulnerabilidade de caminhoneiros ao HIV/aids, os estudos de cunho sociocultural $^{29,30}$ e alguns dos estudos avaliativos ${ }^{35,36}$ destacaram-se por contribuições significativas à análise da vulnerabilidade, pois não se restringiram à dimensão individual, mas ampliaram o leque analítico para questões da vulnerabilidade social e programática. Estes estudos situaram a problemática para além do comportamentalismo, aproximando-se, portanto, da análise de dimensões do conceito de vulnerabilidade.

Por exemplo, o estudo realizado por Sastry ${ }^{30}$, ao analisar narrativas de caminhoneiros em rodovias indianas, mostrou que estes sujeitos vivenciam um contexto de vulnerabilidade social intenso, marcado pela marginalização, informalidade laboral, violência e condições precárias de trabalho. O autor argumenta que apesar de muitos artigos terem sido publicados ressaltando a importância dos fatores estruturais para o risco de infecção pelo HIV, as pesquisas sobre os caminhoneiros, realizadas neste país, ignoram os efeitos do contexto cultural, social e econômico no processo do risco. Através da análise das narrativas dos sujeitos, o autor argumenta que o risco de infecção pelo HIV está situado dentro da opressão estrutural, que é operada pela pobreza e destruição das ocupações laborais tradicionais. Em outro artigo ${ }^{31}$, com base na mesma pesquisa etnográfica, o autor aborda as questões sociais, culturais e estruturais que podem influenciar o uso do preservativo, como por exemplo, a disponibilidade e o custo do preservativo e o prazer sexual como maneira de desviar a atenção das más condições de trabalho.

Em Uganda, a pesquisa realizada por Gysels et al. ${ }^{29}$ sobre a cultura sexual de caminhoneiros, intermediários ("cafetões") e profissionais do sexo também trouxe importante contribuição à análise de vulnerabilidade, pois revelou uma complexa rede de significados sobre os serviços sexuais. Nesta pesquisa, a maioria dos caminhoneiros investigados fazia uso do serviço de intermediários na negociação de serviço sexual, que funcionavam tanto como intérpretes da língua local, quanto como uma garantia de relaciona- 
mento sexual com proteção de roubos e, até mesmo, de doenças. A crença de que os intermediários conhecem as mulheres "seguras" garantiria, portanto, a segurança sexual e física dos caminhoneiros daquela região estudada.

Alguns estudos avaliativos também estiveram mais próximos da análise de vulnerabilidade. $\mathrm{O}$ estudo desenvolvido por Schneider et al. ${ }^{36}$ examinou o compromisso inicial para com a Profilaxia Pré-Exposição (PrEP) e circuncisão entre caminhoneiros através do AIDS Risk Reduction Model. Este estudo, embora tivesse abordado questões sobre comportamentos de risco, não limitou o escopo de análise a estas questões, mas realizou também o escrutínio de normas sociais e religiosas que poderiam influenciar as diversas decisões sexuais e as percepções acerca da circuncisão.

Nesta categoria de estudos, destacou-se também o estudo de Witte et al. ${ }^{35}$, que avaliou a eficácia de materiais de campanha contra a aids no Kenya entre grupos de caminhoneiros e seus assistentes, bem como de profissionais do sexo e homens jovens. Os principais resultados que os autores demonstraram foi que em populações com alta percepção de risco de infecção pelo HIV (como no caso dos caminhoneiros), os panfletos que mostravam as estratégias de prevenção eram mais eficazes do que os cartazes que focavam excessivamente na ameaça do vírus.

Observou-se a presença de poucos estudos qualitativos publicados que investigassem a temática da vulnerabilidade de caminhoneiros ao HIV/aids. Este fato suscita a hipótese de que os estudos qualitativos ainda possuem um lugar marginal na produção de dados no campo da saúde pública sobre a temática. Além disso, chama-se atenção para alguns artigos que utilizaram o método qualitativo apenas para suplementação de informações para dados quantitativos, sem aprofundamento teórico-reflexivo, como por exemplo os estudos realizados no Paquistão ${ }^{44}$ e na Croácia ${ }^{43}$. Apesar das críticas apontadas, os estudos qualitativos aqui revisados trouxeram muitas contribuições ao campo das investigações sobre HIV/aids, principalmente por aprofundar e resgatar questões relevantes que outra metodologia não abordaria.

A maioria dos estudos apresentou a população de caminhoneiros como um grupo social em um contexto de maior vulnerabilidade ao HIV/ aids, e demonstrou principalmente aspectos individuais, majoritariamente comportamentais, tais como a prática do sexo desprotegido com diversos tipos de parcerias sexuais ao longo das estradas (com destaque para as profissionais do sexo). Este fato, portanto, poderia justificar a devassa, realizada por muitos estudos, dos vários aspectos sociais e culturais relacionados à sexualidade e às práticas sexuais dos caminhoneiros, bem como a intervenção de serviços de prevenção de HIV e sua avaliação (clínicas noturnas, PrEP, circuncisão, materiais de campanha). Entretanto, apesar da maioria dos trabalhos publicados terem populações de países pouco desenvolvidos e desiguais, pouco espaço é reservado à análise da vulnerabilidade socioestrutural em que estão submetidos estes trabalhadores, principalmente o que se refere aos contextos do processo de trabalho. Existe, portanto, uma lacuna importante na literatura sobre a relação entre estes contextos estruturais e a epidemia de HIV/aids.

Sabe-se que o conceito de vulnerabilidade não se limita à gênese do agravo, mas engloba a interação entre agente, hospedeiro e meio ${ }^{7}$. E também considera que as características do processo saúde-doença são intrinsecamente determinadas pelo cenário dos serviços de saúde, incluindo os seus saberes e tecnologias. A partir deste pressuposto, pode-se afirmar que as ações de saúde não são apenas soluções, mas também parte do problema a ser resolvido, isto é, "os problemas de saúde são, portanto, sempre processos saúde-doença-cuidado"7.

Portanto, acreditamos que o conceito de vulnerabilidade poderá, ainda na vigência da meta 90-90-903 , oferecer subsídios para a compreensão da complexidade dos diversos fatores envolvidos no processo de saúde-doença-cuidado, porque oportuniza a integração de conhecimentos de áreas distintas em um grande esforço de compreensão dos quadros mais amplos em que as práticas e realidades sociais especificamente vividas pelos sujeitos em situação de vulnerabilidade se estruturam. Tal esforço poderá colaborar para o entendimento das complexas relações existentes entre fatores individuais, programáticos e sociais, responsáveis pela intensificação da vulnerabilidade dos sujeitos ao HIV/aids. 


\section{Agradecimentos}

Agradeço ao professor Marcelo Eduardo Pfeiffer Castellanos, orientador da minha dissertação de mestrado no Instituto de Saúde Coletiva, por provocar diversas reflexões em torno do conceito de vulnerabilidade.

\section{Referências}

1. Joint United Nations Programme on HIV/AIDS (UNAIDS). Fact Sheet 2016 Global Statistics - 2015 Regional Statistics - 2015. Geneva: UNAIDS; 2016. p. 8-13.

2. Brasil. Boletim Epidemilógico HIV/AIDS. Bol Epidemiológico HIVAIDS 2015; p. 1-100.

3. Joint United Nations Programme on HIV/AIDS (UNAIDS). The gap report; 2014. [cited 2013 Nov 5]. Available from: http://www.unaids.org/en/media/unaids/ contentassets/documents/unaidspublication/2014/ UNAIDS_Gap_report_en.pdf

4. Paiva V, Ferguson L, Aggleton P, Mane P, Kelly-Hanku A, Giang le M, Barbosa RM, Caceres CF, Parker R. O estado atual da pesquisa sobre as dimensões sociais políticas e legais do HIV Estado actual de la investigación sobre las dimensiones sociales, políticas y le. Cad Saude Publica 2015; 31(3):477-486.

5. Aggleton P, Parker R. Moving Beyond Biomedicalization in the HIV Response: Implications for Community Involvement and Community Leadership Among Men Who Have Sex with Men and Transgender People. Am J Public Health 2015; 105(8):e1-7.

6. Herzlich C, Pierret J. Uma doença no espaço público. A AIDS em seis jornais franceses. Physis Rev Saude Coletiva 2005; 15(Supl.):71-101.

7. Ayres JR, Paiva V, França-Júnior I. Conceitos e práticas de prevenção: da história natural da doença ao quadro da vulnerabilidade e direitos humanos. In: Paiva V, Ayres JR, Buchalla C, organizadores. Vulnerabilidade e direitos humanos - prevenção e promoção da saúde livro I, da doença à cidadania. Curitiba: Juruá; 2012. p. 71-94.

8. Garcia S, Souza FM. Vulnerabilidades ao HIV/aids no Contexto Brasileiro: Iniquidades de gênero, raça e geração. Saude e Soc 2010; 19(Supl. 2):9-20.

9. Botão C, Horth RZ, Frank H, Cummings B, Inguane C, Sathane I, McFarland W, Fisher Raymond H, Young PW. Prevalence of HIV and Associated Risk Factors Among Long Distance Truck Drivers in Inchope, Mozambique, 2012. AIDS Behav 2015; 20(4):811-820.

10. Kiderlen TR, Conteh M, Roll S, Seeling S, Weinmann S. Cross-sectional study assessing HIV-related knowledge, attitudes and behavior in the Namibian truck transport sector: Readjusting HIV prevention programs in the workplace. J Infect Public Health [Internet]. 2015 [cited 2013 Nov 5]; 8(4):346-354. Available from: http://dx.doi.org/10.1016/j.jiph.2015.02.001

11. Azuonwu O, Erhabor O, Frank-Peterside N. HIV infection in long-distance truck drivers in a low income setting in the Niger Delta of Nigeria. J Community Health 2011; 36(4):583-587.
12. Yaya I, Landoh DE, Saka B, Vignikin K, Aboubakari A-S, N'dri KM, Gbetoglo KD, Edorh AM, Ahlegnan K, Yenkey HC, Toudeka AS, Pitché P. Consistent Condom Use during Casual Sex among Long-Truck Drivers in Togo. PLoS One [Internet]. 2016 [cited 2013 Nov 5]; 11(4):e0153264. Available from: http://dx.plos. org/10.1371/journal.pone.0153264

13. Zhang X, Chow EPF, Wilson DP, Sun X, Zhao R, Zhang J, Jing J, Zhang L. Prevalence of HIV and syphilis infections among long-distance truck drivers in China: A data synthesis and meta-analysis. Int J Infect Dis 2013; 17(1):2-7.

14. Pandey A, Benara SK, Roy N, Sahu D, Thomas M, Joshi DK, Sengupta U, Paranjape RS, Bhalla A, Prakash A; IBBA Study Team. Risk behaviour, sexually transmitted infections and HIV among long-distance truck drivers: a cross-sectional survey along national highways in India. AIDS 2008; 22(Supl. 5):S81-90.

15. Bhatnagar T, Sakthivel Saravanamurthy P, Detels R. Sexual Behaviors and Partner-Specific Correlates of Heterosexual Anal Intercourse Among Truck Drivers and Their Wives in South India. Arch Sex Behav 2014; 44(2):295-306.

16. Sawal N, Hans GDR, Verma G. Sexual practices, myths and misconceptions among long distance truck drivers in North India. Qjm 2016; 109(7):467-472.

17. Masson VA, Monteiro MI. Vulnerabilidade à Doenças Sexualmente Transmissíveis/AIDS e uso de drogas psicoativas por caminhoneiros. Rev Bras Enferm 2010; 63(1):79-83.

18. Leal AF. No peito e na raça " - a construção da vulnerabilidade de caminhoneiros : um estudo antropológico de políticas públicas [tese]. Porto Alegre: UFRGS; 2008.

19. Malta M, Bastos FI, Pereira-Koller EM, Cunha MD, Marques C, Strathdee S. A qualitative assessment of long distance truck drivers' vulnerability to HIV/AIDS in Itajaí, southern Brazil. AIDS Care 2006; 18(5):489-496.

20. Teles SA, Matos MA, Caetano KAA, Costa LA, França DDS, Pessoni GC, Brunini SM, Martins RMB. Comportamentos de risco para doenças sexualmente transmissíveis em caminhoneiros no Brasil. Rev Lat-Am Saude Pub 2008; 24(1):25-30.

21. Ferreira LOC, Oliveira ES, Raymond HF, Chen SY, McFarland W. Use of time-location sampling for systematic behavioral surveillance of truck drivers in Brazil. AIDS Behav 2008; 12(Supl. 1):32-38.

22. Faria KR, Karine T, Ferreira DA, Elisa M, Almeida F, Guedes HM. Comportamentos de risco quanto ao Vírus da Imunodeficiência Humana entre caminhoneiros. Rev. enferm.UERJ 2015; 23(1):27-32. 
23. Knauth DR, Leal AF, Pilecco FB, Seffner F, Teixeira MAB. Manter-se acordado: a vulnerabilidade dos caminhoneiros no Rio Grande do Sul. Rev Saude Publica 2012; 46(5):886-893.

24. Ulhôa MA, Marqueze EC, Lemos LC, Silva LG, Silva AA, Nehme P, Fischer FM, Moreno CRC. Distúrbios psíquicos menores e condições de trabalho em motoristas de caminhão Minor psychiatric disorders and working conditions in truck drivers. Rev Saude Publica 2010; 44(6):1130-1136.

25. Jora NP, Magalhães TR, Domingos JBC, Pillon SC. Campanha saúde na estrada: avaliação do padrão de consumo de álcool e do estresse. Rev. Eletr. Enf. 2010; 12(1):37-46.

26. Whittemore R, Knafl K. The integrative review: Updated methodology. J Adv Nurs 2005; 52(5):546-553.

27. Ganong LH. Integrative reviews of nursing research. Res Nurs Heal 1987; 10(1):1-11.

28. Moher D, Liberati A, Tetzlaff J, Altman DG, Grp P. Preferred Reporting Items for Systematic Reviews and Meta-Analyses: The PRISMA Statement (Reprinted from Annals of Internal Medicine). Phys Ther 2009; 89(9):873-880.

29. Gysels M, Pool R, Bwanika K. Truck drivers, middlemen and commercial sex workers: AIDS and the mediation of sex in south west Uganda. AIDS Care 2001; 13(3):373-385.

30. Sastry S. Long Distance Truck Drivers and the Structural Context of Health: A Culture-Centered Investigation of Indian Truckers' Health Narratives. Health Commun [Internet]. 2015 [cited 2013 Nov 5]; (August):1-12. Available from: http://www.tandfonline.com/doi/full/ 10.1080/10410236.2014.947466

31. Sastry S. Structure and agency in long-distance truck drivers' lived experiences of condom use for HIV prevention. Cult Health Sex [Internet]. 2016 [cited 2013 Nov 5]; 18(5):553-566. Available from: http:// search.ebscohost.com/login.aspx?direct $=$ true $\& \mathrm{db}=\mathrm{jl}$ h\&AN=114015484\&site=ehost-live

32. Nyamwaya D. Anthropology and HIV/AIDS prevention in Kenya. AIDS Soc 1993; 4(4):4.

33. Walden VM, Mwangulube K, Makhumula-Nkhoma P. Measuring the impact of a behaviour change intervention for commercial sex workers and their potential clients in Malawi. Health Educ Res [Internet]. 1999 Aug [cited 2013 Nov 5]; 14(4):545-554. Available from: http://www.ncbi.nlm.nih.gov/pubmed/10557524

34. Lafort Y, Geelhoed D, Cumba L, Lázaro CDDM, Delva W, Luchters S, Temmerman M. Reproductive health services for populations at high risk of HIV: Performance of a night clinic in Tete province, Mozambique. BMC Health Serv Res [Internet]. 2010 Jan [cited 2013 Nov 5]; 10:144. Available from: http://www.pubmedcentral. nih.gov/articlerender.fcgi? artid=2890643\&tool=pmcentrez\&rendertype $=$ abstract

35. Witte KIM, Cameron KA, Lapinski MK, Nzyuko S. A theoretically based evaluation of HIV/AIDS prevention campaigns along the trans-Africa highway in Kenya. $J$ Heal Commun 1998; 3(4):345-363.

36. Schneider J, Dandona R, Pasupneti S, Lakshmi V, Liao C, Yeldandi V, Mayer K. Initial commitment to pre-exposure prophylaxis and circumcision for HIV prevention amongst Indian truck drivers. PLoS One [Internet]. 2010 Jan [cited 2013 Nov 5]; 5(7):e11922. Available from: http://www.pubmedcentral.nih.gov/articlerender.fcgi?artid=2912853\&tool=pmcentrez\&rendertype $=$ abstract
37. Gibney L, Saquib N, Metzger J. Behavioral risk factors for STD/HIV transmission in Bangladesh's trucking industry. Soc Sci Med [Internet]. 2003 Apr [cited 2013 Nov 5]; 56(7):1411-1424. Available from: http://linkinghub.elsevier.com/retrieve/pii/S0277953602001387

38. Ntozi JPM, Najjumba IM, Ahimbisibwe F, Ayiga N, Odwee J. Has the HIV/AIDS epidemic changed sexual behaviour of high risk groups in Uganda? Afr Health Sci [Internet]. 2003 Dec [cited 2013 Nov 5]; 3(3):107116. Available from: http://www.pubmedcentral.nih. gov/articlerender.fcgi?artid=2141609\&tool=pmcentrez\&rendertype $=$ abstract

39. Sousa LMS, Silva LS, Palmeira AT. Representações sociais de caminhoneiros de rota curta sobre HIV/ AIDS. Psicol Soc [Internet]. 2014 [cited 2013 Nov 5]; 26(2):346-355. Available from: http://www.scielo.br/ pdf/psoc/v26n2/a11v26n2.pdf

40. Mtenga S, Shamba D, Wamoyi J, Kakoko D, Haafkens J, Mongi A, Kapiga S, Geubbels E. How long-distance truck drivers and villagers in rural southeastern Tanzania think about heterosexual anal sex: a qualitative study. Sex Transm Infect 2015; 91(8):576-580.

41. Sri Krishnan AK, Hendriksen E, Vallabhaneni S, Johnson SL, Raminani S, Kumarasamy N, Hobsen J, Solomon S, Mayer KH, Safren SA. Sexual behaviors of individuals with HIV living in South India: A qualitative study. AIDS Educ Prev 2007; 19(4):334-345.

42. Stratford D, Ellerbrock TV, Akins JK, Hall HL. Highway cowboys, old hands, and Christian truckers: Risk behavior for human immunodeficiency virus infection among long-haul truckers in Florida. Soc Sci Med 2000; 50(5):737-749.

43. Baćak V, Soh D. On the road: Croatian truck drivers, commercial sex and HIV/AIDS. Coll Antropol [Internet]. 2006 [cited 2013 Nov 5]; 30(Supl. 2):99103. Available from: http://www.ncbi.nlm.nih.gov/ pubmed/17508482

44. Agha S. Sexual behaviour among truck drivers in Pakistan. Cult Health Sex 2002; 4(2):191-206.

45. Mann JTD. From epidemiology to vulnerability to human rights. In: Mann JTD, editor. AIDS in the world II. New York: Oxford University; 1996. p. 427-476.

46. Parker R, Camargo Júnior KR. Pobreza e HIV/AIDS: aspectos antropológicos e sociológicos. Cad Saude Publica 2000; 16(Supl. 1):89-102.

47. Minayo MC. O desafio do conhecimento: pesquisa qualitativa em saúde. São Paulo: Hucitec; 2007.

48. Czeresnia D. Do contágio à transmissão. Ciência e cultura na gênese do conhecimento epidemiológico. Rio de Janeiro: Editora Fiocruz; 1997.

49. Ayres JRDCM, Paiva V, França I, Gravato N, Lacerda R, Della Negra M, Marques HH, Galano E, Lecussan P, Segurado AC, Silva MH. Vulnerability, human rights, and comprehensive health care needs of young people living with HIV/AIDS. Am J Public Health [Internet]. 2006 Jun [cited 2013 Oct 17]; 96(6):1001-1006. Available from: http://www.pubmedcentral.nih.gov/articlerender.fcgi?artid=1470608\&tool=pmcentrez\&rendertype $=$ abstract

Artigo apresentado em 27/05/2016

Aprovado em 16/03/2017

Versão final apresentada em 18/03/2017 